\title{
Pediatric asthma control during the COVID-19 pandemic: a systematic review and meta-analysis
}

\author{
Ze Yang ${ }^{1}$, Xiang Wang ${ }^{1}$, Xigang Wan ${ }^{1}$, Menglei Wang ${ }^{1}$, Zonghua Qiu ${ }^{1}$, Jiali Chen ${ }^{1}$, \\ Manhao Shi ${ }^{1}$, Shiyi Zhang ${ }^{1}$, and Yongliang Xia ${ }^{1}$ \\ ${ }^{1}$ Zhejiang Chinese Medical University
}

July 22, 2021

\begin{abstract}
Background: During the current ongoing COVID-19 pandemic, studies had reported that patients with asthma would experience increased asthma-associated morbidity because of the respiratory virus SARS-CoV-2 infection, based on experience with other respiratory viral infections. However, some studies suggested that there was no apparent increase in asthma related morbidity in children with asthma, it is even possible that due to reduced exposures due to confinement, such children may have improved outcomes. In order to understand the impact of Covid-19 on asthma control in children, we performed this systematic review and meta-analysis. Methods: We searched PubMed, Embase, and Cochrane Library to find literature from December 2019 to June 2021 related to Covid-19 and children's asthma control, among which results such as abstracts, comments, letters, reviews and case reports were excluded. The level of asthma control during the COVID-19 pandemic was synthesized and discussed. Results: A total of 20456 subjects were included in 7 studies. Random effect model is used to account for the data. Compared to the same period before the COVID-19 pandemic, asthma exacerbation, asthma admission, emergency room visit reduced a lot. The outcome of use of inhaled corticosteroids and Beta-2 agonists shows no significant difference. Conclusion: Compared to the same period before the COVID-19 pandemic and the measures in response to it, the level of asthma control has been significantly improved. We need to understand the exact factors leading to these improvements and find methods to sustain it.
\end{abstract}

\section{Title Page}

Title: Pediatric asthma control during the COVID-19 pandemic: a systematic review and meta-analysis

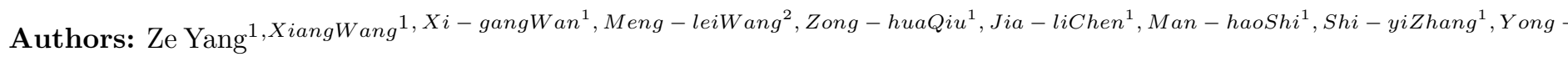

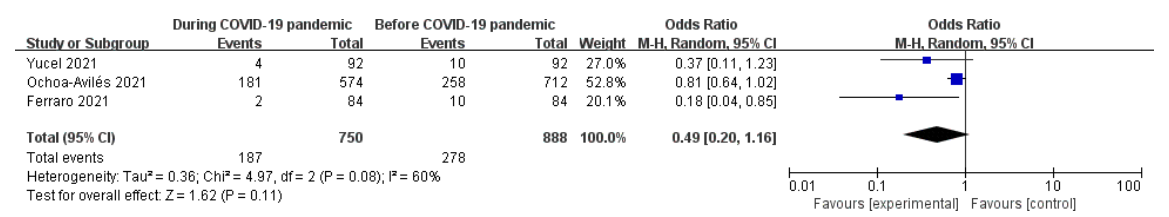

Figure 2 Forest plots for comparison of asthma exacerbation between COVID-19 pandemic and before it. 


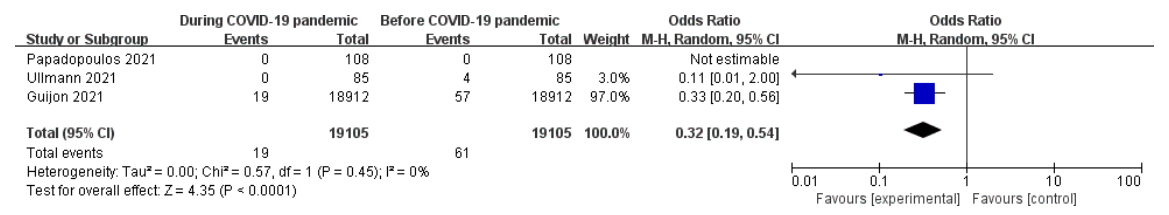

Figure 3 Forest plots for comparison of asthma admission between COVID-19 pandemic and before it.

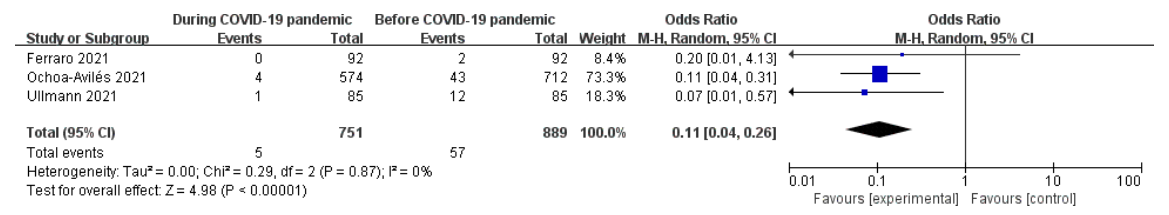

Figure 4 Forest plots for comparison of emergency room visit between COVID-19 pandemic and before it.

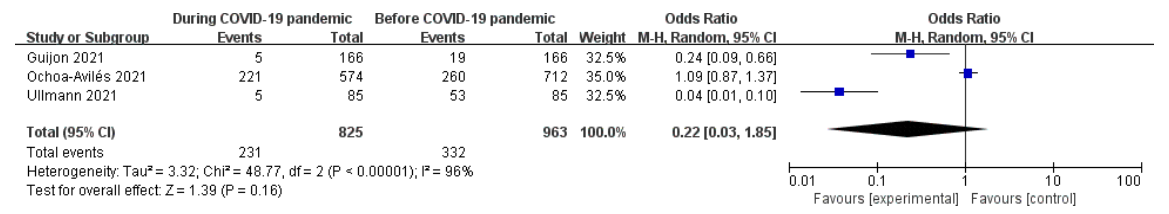

Figure 5 Forest plots for comparison of use of inhaled corticosteroid between COVID-19 pandemic and before it.

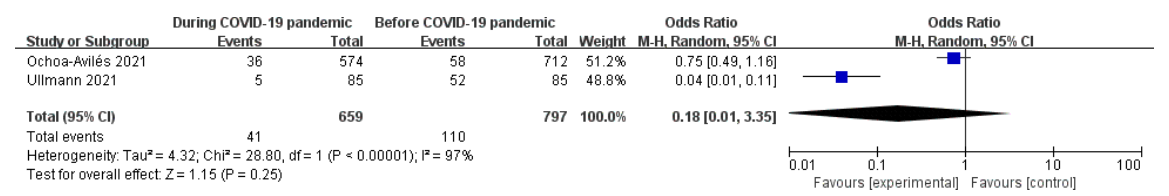

Figure 6 Forest plots for comparison of use of Beta-2 agonists between COVID-19 pandemic and before it. Hosted file

Table 1.docx available at https://authorea.com/users/427123/articles/531377-pediatric-asthmacontrol-during-the-covid-19-pandemic-a-systematic-review-and-meta-analysis 